\title{
Space-filling branes and gaugings
}

\author{
Fabio Riccioni* \\ INFN Sezione di Roma \\ Dipartimento di Fisica \\ Università di Roma "La Sapienza" \\ Piazzale Aldo Moro 2 \\ 00185 Roma (Italy) \\ E-mail: Fabio.Riccionieromal.infn.it
}

\begin{abstract}
We consider a $T^{6} /\left[\mathbb{Z}_{2} \times \mathbb{Z}_{2}\right]$ type-IIB O3-O7 orientifold model with all the possible non-geometric fluxes included. We show that the tadpole conditions can all be cancelled by including spacefilling branes which are all compatible with supersymmetry. We discuss the exotic nature of most of these branes, and show how they naturally couple to mixed-symmetry potentials. We then move to discuss gauged supergravity theories in any dimension, and show that in general if one performs a $\mathbb{Z}_{2}$ truncation that halves the amount of supersymmetry, the quadratic constraints that survive this truncation but are not required by the supersymmetry of the truncated theory are always in one-to-one correspondence with the space-filling branes that all preserve the same supersymmetry of such theory. This is an overview of the results published in references [1] and [2].
\end{abstract}

Corfu Summer Institute 2018 "School and Workshops on Elementary Particle Physics and Gravity" (CORFU2018)

31 August - 28 September, 2018

Corfu, Greece

*Speaker. 


\section{Introduction}

Fluxes play a crucial role in string theory because of their relevance in moduli stabilisation (for a review, see e.g. [3]). Considering in particular O3-O7 orientifold type-IIB Calabi-Yau compactifications with NS-NS and RR 3 -form fluxes $\mathscr{H}_{3}$ and $\mathscr{F}_{3}$ turned on, the $\mathscr{N}=1$ classical superpotential is given by [4]

$$
W_{\mathrm{IIB} / \mathrm{O} 3}=\int\left(\mathscr{F}_{3}-i S \mathscr{H}_{3}\right) \wedge \Omega
$$

where $\Omega$ is the holomorphic 3-form of the Calabi-Yau manifold and $S$ is the axion-dilaton. On the other hand, the presence of the Chern-Simons term

$$
\int C_{4} \wedge \mathscr{H}_{3} \wedge \mathscr{F}_{3}
$$

in the IIB supergravity theory implies that the NS-NS and RR fluxes act as sources for the RR 4form potential $C_{4}$. This source has to be cancelled for consistency, implying that fluxes are suitably quantised so that the source can be compensated by changing the net amount of D3-branes in the orientifold theory.

We will focus on the specific case of a $T^{6} /\left[\mathbb{Z}_{2} \times \mathbb{Z}_{2}\right]$ IIB O3-O7 orientifold. Factorising the 6-torus as $T^{6}=\bigotimes_{i=1}^{3} T_{(i)}^{2}$, the two $\mathbb{Z}_{2}$ 's act as $(-1,-1,1)$ and $(1,-1,-1)$ respectively on the coordinates $\left(x^{i}, y^{i}\right)$ of the three 2-tori. Following the notation of [5] (see also [6, 7, 8, 9]) we define the three 2-forms $\omega_{i}=-d x^{i} \wedge d y^{i}$ as the natural basis for closed 2-forms, while the basis for closed 4-forms is given by the Hodge duals $\tilde{\omega}_{i}=* \omega_{i}$. The Kähler form $J$ and the holomorphic 3 -form $\Omega$ are given by the expressions

$$
J=\sum_{i=1}^{3} A_{i} \omega_{i} \quad \Omega=\left(d x^{1}+i \tau_{1} d y^{1}\right) \wedge\left(d x^{2}+i \tau_{2} d y^{2}\right) \wedge\left(d x^{3}+i \tau_{3} d y^{3}\right)
$$

where $A_{i}$ and $\tau_{i}$ are the volumes and complex structure moduli of the three different tori. The O3O7 orientifold projection acts like $\Omega_{P}(-1)^{F_{L}} \sigma_{B}$, where $\Omega_{P}$ is the world-sheet parity reversal, $F_{L}$ is the world-sheet left-mover fermionic number and $\sigma_{B}$ is the space-time involution acting on the coordinates as

$$
\sigma_{B}\left(x^{i}\right)=-x^{i} \quad \sigma_{B}\left(y^{i}\right)=-y^{i} .
$$

The untwisted moduli ${ }^{1}$ are the axion-dilaton $S$, the complex-structure moduli $U_{i}$ that simply coincide with the toroidal complex structures, i.e. $U_{i}=\tau_{i}$, and the complex Kähler moduli $T_{i}$ which are given in terms of the Kähler form and the RR 4-form by the expression

$$
\mathscr{J}_{\mathrm{c}}=C_{4}+\frac{i}{2} e^{-\phi} J \wedge J=i \sum_{i} T_{i} \tilde{\omega}_{i}
$$

These seven moduli parametrise the space $(\mathrm{SL}(2, \mathbb{R}) / \mathrm{SO}(2))^{7}$, and the theory possesses a U-duality symmetry $\operatorname{SL}(2, \mathbb{Z})$. One can then turn on $\mathscr{H}_{3}$ and $\mathscr{F}_{3}$ fluxes with indices on the three different tori. This makes in total 16 flux components, and by plugging this into eq. (1.1) using eq. (1.3) one

\footnotetext{
${ }^{1}$ One can construct freely acting $\mathbb{Z}_{2} \times \mathbb{Z}_{2}$ orbifolds [10] such that the twisted sector does not contain massless scalars. Orientifolds of freely-acting orbifolds have been constructed in e.g. [11, 12, 13]. For a review on orientifold models, see e.g. [14].
} 
obtains a superpotential which is at most cubic in the $U$ moduli and linear in $S$, with no dependence on the $T$ moduli.

Before turning on fluxes, one can perform three T-dualities, each along a cycle of a different torus, to map the model to the $T^{6} /\left[\mathbb{Z}_{2} \times \mathbb{Z}_{2}\right]$ IIA O6 orientifold. In particular, if one T-dualises along the $x^{i}$ directions, the orientifold involution $\sigma_{A}$ acts on the coordinates as

$$
\sigma_{A}\left(x^{i}\right)=x^{i} \quad \sigma_{A}\left(y^{i}\right)=-y^{i},
$$

and the $\mathrm{O} 6$ planes are localised in the $y^{i}$ directions. When fluxes are turned on, one obtains the superpotential of the IIA theory by acting with three T-dualities along the $x$ 's on the fluxes of the IIB theory. In particular, given that in general the NS-NS 3-form flux $\mathscr{H}_{a b c}$ is mapped to the metric flux $f_{a b}^{c}$ and to the non-geometric $\mathscr{Q}_{a}^{b c}$ and $\mathscr{R}^{a b c}$ fluxes according to the chain of rules [6] (see also [15])

$$
\mathscr{H}_{a b c} \stackrel{\mathrm{T}_{c}}{\longleftrightarrow} f_{a b}^{c} \stackrel{\mathrm{T}_{b}}{\longleftrightarrow} \mathscr{Q}_{a}^{b c} \stackrel{\mathrm{T}_{a}}{\longleftrightarrow} \mathscr{R}^{a b c},
$$

one finds that mirror symmetry can only be restored if one allows the presence of non-geometric fluxes. Moreover, in the IIA theory the orientifold projections also allows for additional flux components that are mapped back in the IIB theory to all the allowed components of the non-geometric flux $\mathscr{Q}_{a}^{b c}$. This gives a total of 24 flux components. The resulting superpotential

$$
W_{\mathrm{IIB} / \mathrm{O} 3}=\int\left(\mathscr{F}_{3}-i S \mathscr{H}_{3}+\mathscr{Q} \cdot \mathscr{J}_{\mathrm{c}}\right) \wedge \Omega
$$

leads to terms linear in the moduli $T_{i}$ and cubic in $U_{i}$.

In the IIB theory one can also include the flux $\mathscr{P}_{a}^{b c}$ which is the S-dual of $\mathscr{Q}_{a}^{b c}$, and find that this flux leads to $S T_{i}$ (times a cubic polynomial in $U_{i}$ ) terms in the $\mathscr{N}=1$ superpotential [5]. In [16] it was shown that the fluxes that are related by T-duality to $\mathscr{P}_{a}^{b c}$ in any dimension are $\mathscr{P}_{a}^{b_{1} . . b_{p}}$ and $\mathscr{P}^{a, b_{1} \ldots b_{p}}$, where $p$ is even in IIB and odd in IIA and the $b$ indices are completely antisymmetrised. Moreover, a universal T-duality rule for this fluxes, analogous to the one in eq. (1.7), was discovered in [17]. This allows to repeat the same argument as before: one can map by mirror symmetry the $\mathscr{P}_{a}^{b c}$ flux of IIB to the $\mathscr{P}$ fluxes of the IIA theory, include all the remaining $\mathscr{P}$ fluxes that are allowed by the IIA orientifold and then map this back to the IIB theory. As a result, one finds that one must also include the 24 components of the IIB flux $\mathscr{P} a, b_{1} \ldots b_{4}$, which leads to terms in the superpotential that are quadratic in $T$ and cubic in $U$.

One can keep using the symmetries of the theory to turn on non-geometric fluxes in the IIA and IIB theories until one finds an expression for the superpotential which is closed under $\mathrm{S}$ and $\mathrm{T}$ dualities [1]. This expression includes all the possible fluxes, and coincides with the one derived using generalised geometry methods in [18]. The resulting superpotential is the most general polynomial which is cubic in the $T$ and $U$ moduli and linear in $S$, and depends on 128 flux components. More precisely, it turns out that these 128 flux components form the $(\mathbf{2}, \mathbf{2}, \mathbf{2}, \mathbf{2}, \mathbf{2}, \mathbf{2}, \mathbf{2})$ representation of $\operatorname{SL}(2, \mathbb{R})^{7}[5]$.

Given that supersymmetry allows all these fluxes to be turned on in the IIB orientifold model, we can ask what are the 3 -brane charges that are induced by them, exactly like the $\mathscr{H}_{3}$ and $\mathscr{F}_{3}$ fluxes generate a charge for the D3-brane. This question was fully answered in [1], and the result is that the tadpole conditions induced by all the fluxes in the $(\mathbf{2}, \mathbf{2}, \mathbf{2}, \mathbf{2}, \mathbf{2}, \mathbf{2}, \mathbf{2})$ belong to 16 irreducible 
representations of $S L(2, \mathbb{R})^{7}$, made of three triplets and four singlets, with the condition that there are either zero or two triplets with respect to the three $S L(2, \mathbb{R})$ 's associated to the $U_{i}$ moduli. One therefore needs for consistency space-filling 3-branes in these representations. What we will discuss in what follows is precisely what these branes are. In particular, we will show that most of these branes are exotic, in the sense that they do not arise from the reduction of branes of the ten-dimensional theory. Moreover, we will show that all these branes are compatible with the supersymmetry preserved in the orientifold model.

One could study what conditions must be imposed on the fluxes to preserve $\mathscr{N}=2$ supersymmetry, i.e. before performing the orientifold projection. In this case one can see that the 6-form $\mathscr{H}_{3} \wedge \mathscr{F}_{3}$ integrated on the internal manifold has to vanish, because otherwise the Chern-Simons term (1.2) would require the inclusion of D3-branes which are not compatible with $\mathscr{N}=2$ supersymmetry. More generally, turning on all possible fluxes one finds for consistency a set of Bianchi identities must be imposed. If one then performs the orientifold projection, and thus keeps only the 128 flux components that survive this projection, it turns out that the Bianchi identities are projected on the 16 representations made of three triplets and four singlets mentioned above.

There is a natural way of reformulating the discussion above in the language of gaugings of supergravity theories. Indeed, from the supergravity viewpoint fluxes are components of the embedding tensor, which characterises the possible gaugings of the theory [19,20]. Supersymmetry imposes a quadratic constraint for the embedding tensor, and one can ask, given a specific $\mathbb{Z}_{2}$ truncation that halves the amount of supersymmetry, whether the quadratic constraints that survive the truncation are actually required by supersymmetry in the truncated theory. It turns out that in general not all of them are needed, and those that can be relaxed are precisely in the representations to which the space-filling branes that preserve precisely the supersymmetry of the truncated theory belong $[21,2]$. Hence, these quadratic constraints can be relaxed provided that one adds the right amount of space-filling branes to cancel the tadpoles. This is precisely what happens in the orientifold model discussed above, but it is actually completely general as we are going to discuss.

The rest of this presentation is organised as follows. In section 2 we will discuss exotic branes in general. In section 3 we will concentrate on space-filling branes in various dimensions, and we will show how one can determine all the branes that in each theory preserve exactly the same supersymmetry. In particular we will show that the space-filling branes required to cancel the tadpoles of the $T^{6} /\left[\mathbb{Z}_{2} \times \mathbb{Z}_{2}\right]$ orientifold model are all compatible with supersymmetry. In section 4 we will move to discuss the relation between space-filling branes preserving the same supersymmetry and quadratic constraints of the embedding tensor. Section 5 will contain some concluding remarks.

\section{Exotic branes}

The prototype example of an exotic brane is the solution that one obtains starting from the NS5-brane solution of either the IIA or the IIB theory and performing two T-dualities along directions transverse to the brane [22]. In order to do this, one has to first smear the NS5-brane along these two directions (say directions 8 and 9), which results in a solution depending only on the two remaining transverse directions 6 and 7, and such that the 2-form $B_{89}$ has a non-trivial monodromy on the 6-7 plane, meaning that

$$
B_{89} \rightarrow B_{89}+1
$$


if one rotates around the brane on the plane. Defining

$$
\rho=B_{89}+i \sqrt{\operatorname{det} G}
$$

where $G$ is the metric in the 8 and 9 directions, one finds that performing two T-dualities in such directions acts on $\rho$ as

$$
\rho \rightarrow-\frac{1}{\rho}
$$

which implies that the resulting solution has a non-trivial momodromy with respect to

$$
-\frac{1}{\rho}=-\frac{B_{89}}{B_{89}^{2}+\operatorname{det} G}
$$

and hence describes a metric that in these directions in not globally defined, i.e. a T-fold [23]. Although the classical solution is a well-defined geometric solution only in eight dimensions, nonetheless T-duality is a symmetry of the quantum theory and therefore we can consider this as a consistent solution of string theory.

This can be generalised to any dimensions. In [24], the $D$ dimensional brane solutions with two transverse directions (so-called defect branes) obtained by performing chains of $\mathrm{S}$ and T-dualities on brane solutions that arise from ten dimensions have been classified. Typically, from the point of view of the ten-dimensional theory most of these solutions are well defined only in the presence of isometries, and from the point of view of the ten-dimensional theory they are non-geometric, in the sense that their monodromy involves the metric in the isometry directions [25]. All branes of this type are dubbed 'exotic' in the literature [26]. Using string dualities, one can derive the tension of all such branes as functions of the string tension and the radii associated to the isometry directions $[27,28]$. What one finds is that for instance the brane of [22] discussed above and related to the NS5-brane by two T-dualities has tension scaling like $g_{s}^{-2} R_{8} R_{9}$. More generally, exotic branes have tensions scaling with positive powers of the isometry directions, which implies that they disappear from the spectrum in the decompactification limit. It is important to notice that by requiring the closure of the brane spectrum under S and T-dualities one obtains not only exotic defect branes, but also exotic branes with one or zero transverse direction, i.e. domain walls and space-filling branes [27, 28]. Moreover, in general one gets powers of the radii higher than one in the formula for the tension. For instance, one can take the D7-brane of IIB in ten dimensions. Under S-duality, this brane is mapped to a 7-brane with tension scaling like $g_{s}^{-3}$, and T-dualising along the two transverse directions 8 and 9 one gets a space-filling brane in eight dimensions with tension scaling like $g_{s}^{-3} R_{8}^{2} R_{9}^{2}$.

In the brane classification of $[29,30,31]$, the non-geometric nature of a particular brane corresponds to the fact that the brane is charged with respect to a mixed-symmetry potential. ${ }^{2}$ In particular, denoting with $A_{p, q, r, .}$ a ten-dimensional mixed-symmetry potential in a representation such that $p, q, r, \ldots$ (with $p \geq q \geq r \ldots$ ) denote the length of each column of its Young Tableau, this corresponds to a brane if some of the indices $p$ are isometries and contain all the indices $q$, which themselves contain all the indices $r$ and so on. The relation between the tension of the exotic brane

\footnotetext{
${ }^{2}$ The mixed-symmetry potentials of the ten-dimensional theories is determined from the infinite-dimensional KacMoody algebra $\mathrm{E}_{11}[32]$.
} 
and its corresponding mixed-symmetry potential is very straightforward: the isometry directions correspond to the repeated indices, and the number of times these indices are repeated is equal to the power dependence on the isometry radius plus one. This means that the 5-brane that one gets performing two T-dualities starting from the NS5-brane couples to a mixed-symmetry potential $A_{8,2}$, while the 7-brane that arises performing two T-dualities starting from the S-dual of the D7-brane couples to a mixed-symmetry potential $A_{10,2,2}$.

The fact that exotic branes couple electrically to mixed-symmetry potentials gives a very simple way to determine how different branes are mapped under T-duality. This is formulated in terms of a universal T-duality rule derived in [17] which states as follows: given a brane whose tension scales like $g_{s}^{-n}$ with respect to the string coupling constant, and associated to a mixed-symmetry potential such that the $a$ index occurs $p$ times (in $p$ different sets of antisymmetric indices), this is mapped by T-duality along $a$ to the brane associated to the potential in which the $a$ index occurs $n-p$ times. Schematically, this can be written as

$$
g_{s}^{-n}: \quad \underbrace{a, a, \ldots, a}_{p} \stackrel{\mathrm{T}_{a}}{\longleftrightarrow} \underbrace{a, a, \ldots, a}_{n-p} .
$$

In the next section we will use this rule to determine all the space-filling branes in any dimension that preserve the same supersymmetry.

\section{Space-filling branes}

A crucial result that applies to all the maximal theories in dimension less than ten is the fact that the 1/2-BPS condition for space-filling branes is degenerate, which means that different branes can preserve the same supersymmetry [33]. This degeneracy was determined in [33] by simply observing that the number of 1/2-BPS space-filling branes is always a multiple of the dimension of the R-symmetry group of the vector central charge $Z_{\mu}$. We can associate to each space-filling brane a $\mathbb{Z}_{2}$ truncation to the half-supersymmetric theory, and given that the degenerate branes all preserve the supersymmetry of the same truncation, we arrive at the obvious conclusion that the number of different supersymmetric $\mathbb{Z}_{2}$ truncations is precisely the dimension of the representation of the central charge $Z_{\mu}$. In this section we want to identify these truncations, and for each truncation we want to identify the branes that are not projected out, i.e. the branes that preserve the same supersymmetry of the truncated theory [2].

We can first consider explicitly the eight-dimensional case. In this case the degeneration of the space-filling branes is equal to 2, and indeed we know that in particular the D7-brane and its S-dual preserve the same supersymmetry $[34,35]$, which is the supersymmetry preserved by the O7 truncation. Performing T-dualities in the compact directions 8 and 9, the D7-brane is mapped to the D9-brane, while the S-dual of the D7-brane is mapped to an exotic space-filling brane, i.e. a brane charged with respect to an 8-form potential whose IIB origin is the mixed-symmetry potential $A_{10,2,2}$. These branes survive the $\mathrm{O} 9$ truncation in eight dimensions. These results can naturally be rephrased in a group theory setting. The global symmetry of the maximal theory is $\operatorname{SL}(3, \mathbb{R}) \times \operatorname{SL}(2, \mathbb{R})$, and the one of the truncated theory is $\mathbb{R}^{+} \times(\operatorname{SL}(2, \mathbb{R}))^{2}$. There are three different truncations, corresponding to the three different ways in which $\operatorname{SL}(2, \mathbb{R})$ can be embedded inside $\mathrm{SL}(3, \mathbb{R})$. The $\mathrm{O} 7$ and $\mathrm{O} 9$ are two of these three truncations , the third being the one in which 
all RR potentials are projected out. The space-filling branes of the maximal theory belong to the 15 of $\operatorname{SL}(3, \mathbb{R})$, and after the truncation this representation is projected on the $\mathbf{3}$ of $\operatorname{SL}(2, \mathbb{R})$. The two 7-branes that preserve the same supersymmetry of the truncation are the two long weights of this representation $[33,36]$.

We then move to considering the seven-dimensional case. In seven dimensions the mixedsymmetry potential $A_{10,2,2}$ gives three different exotic 7-branes, one with isometries in the directions 8 and 9, one in the directions 7 and 9 and finally one in the directions 7 and 8. Obviously all these branes preserve the same supersymmetry because each of them preserves the same supersymmetry of the D9-brane. If we now perform two T-dualities, say along the directions 8 and 9 , the D9 goes back to the D7 wrapping the direction 7 and the exotic brane with isometries along 8 and 9 goes back to the S-dual of the D7, again wrapping the direction 7 . On the other hand, the other two exotic branes are mapped to two additional exotic branes with tensions $g_{s}^{-3} R_{8} R_{7}^{2}$ and $g_{s}^{-3} R_{9} R_{7}^{2}$, corresponding to two different components of the mixed-symmmetry potential $A_{9,2,1}$. The conclusion is that in seven dimensions in the cases of both the $\mathrm{O} 7$ and the $\mathrm{O} 9$ truncations there are 4 space-filling branes preserving the same supersymmetry of the truncation. As in eight dimensions, we can understand this from group theory. In this case the symmetry of the maximal theory is $\operatorname{SL}(5, \mathbb{R})$, which is reduced to $\mathbb{R}^{+} \times \operatorname{SL}(4, \mathbb{R})$ by the $\mathbb{Z}_{2}$ truncation to the half-maximal theory. There are five ways of performing this truncation. The 7-branes of the maximal theory belong to the 70 of $\operatorname{SL}(5, \mathbb{R})$, which is projected on the 10 of $\operatorname{SL}(4, \mathbb{R})$. The four 7-branes that preserve the same supersymmetry of the truncation correspond to the four long weights of the $\mathbf{1 0}$.

The analysis can be performed for the maximal theories in all dimensions. The results are shown in the first two columns of table 1, where the red representations contain the branes that preserve the same supersymmetry of the truncated theory. In four dimensions there are 63 different $\mathbb{Z}_{2}$ truncations of the maximal theory to the half-maximal one, with global symmetry $\operatorname{SL}(2, \mathbb{R}) \times$ $\mathrm{SO}(6,6)$. As the table shows, the space-filling 3-branes that preserve the same supersymmetry of the truncation belong to the $(\mathbf{1}, \overline{\mathbf{4 6 2}})$. There are 32 such branes, corresponding to the long weights of this representation. We can identify 32 of the 63 truncations as the O9 truncation, the O3 truncation, the $15 \mathrm{O} 5$ truncations and the $15 \mathrm{O} 7$ truncations. One can show that for each of these truncations the heaviest branes at weak coupling are exotic branes with tension scaling like $g_{s}^{-7}$ [2]. The remaining truncations are related to these by chains of $\mathrm{S}$ and T-dualities.

As the table shows, starting from six dimensions, apart from the branes that preserve the same supersymmetry of the truncation, there are additional space-filling branes that are not projected out by the $\mathbb{Z}_{2}$ truncation but are 1/2-BPS branes of the truncated theory. This is consistent with the fact that such theories can be further truncated to quarter-maximal theories. One can then repeat the same analysis and determine the space-filling branes among these 1/2-BPS branes that preserve the same supersymmetry of the truncation to the quarter-maximal theory. The results are summarised in the remaining columns of table 1.

All this analysis allows to finally answer the question: in the $T^{6} /\left[\mathbb{Z}_{2} \times \mathbb{Z}_{2}\right]$ IIB O3-O7 orientifold model, what are the branes that preserve the same supersymmetry of the O3-O7 truncation to $\mathscr{N}=1$, and what is their relation with the tadpole conditions induced by the fluxes? Out of the 15 different $\mathrm{O} 7$ truncations of the maximal theory, corresponding to D7-branes wrapping the 15 4-cycles of $T^{6}$, in the orbifold there are three truncations that survive, corresponding to D7-branes wrapping two of the three tori of the orbifold. In the truncation from $\mathscr{N}=2$ to $\mathscr{N}=1$, these 


\begin{tabular}{|c|c|c|c|c|}
\hline$D$ & 32 & 16 & 8 & 4 \\
\hline \multirow{2}{*}{8} & $\operatorname{SL}(3, \mathbb{R}) \times \operatorname{SL}(2, \mathbb{R})$ & $\mathbb{R}^{+} \times(\operatorname{SL}(2, \mathbb{R}))^{2}$ & & \\
\hline & $(15,1)$ & $(\mathbf{3}, \mathbf{1})$ & & \\
\hline \multirow{2}{*}{7} & $\operatorname{SL}(5, \mathbb{R})$ & $\mathbb{R}^{+} \times \operatorname{SL}(4, \mathbb{R})$ & & \\
\hline & 70 & 10 & & \\
\hline \multirow{6}{*}{6} & \multirow{2}{*}{$\mathrm{SO}(5,5)$} & $\mathbb{R}^{+} \times \mathrm{SO}(4,4)$ & \multirow{2}{*}{$\mathbb{R}^{+} \times \mathrm{SO}(4,4)$} & \\
\hline & & $\mathrm{SO}(5,5)$ & & \\
\hline & \multirow{2}{*}{320} & $35_{\mathrm{V}} \quad 35_{\mathrm{V}}$ & $35_{\mathrm{V}}$ & \\
\hline & & 320 & $\mathbf{3 5} 5_{\mathrm{V}} \oplus \mathbf{3 5} \mathrm{V}_{\mathrm{V}}$ & \\
\hline & \multirow{2}{*}{$\overline{126}$} & $\mathbf{3 5} \mathbf{S}_{\mathrm{S}} \oplus \mathbf{3 5}_{\mathrm{C}}$ & $35_{\mathrm{S}} \oplus 35_{\mathrm{C}}$ & \\
\hline & & $\overline{\mathbf{1 2 6}}$ & & \\
\hline \multirow[b]{2}{*}{5} & $E_{6(6)}$ & $\overline{\mathbb{R}^{+} \times \mathrm{SO}(5,5)}$ & 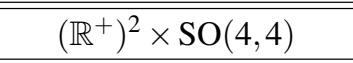 & \\
\hline & $\overline{1728}$ & $\begin{array}{cc}\overline{126} & 320 \\
210\end{array}$ & $\begin{array}{l}35_{\mathrm{V}} \oplus 35_{\mathrm{V}} \\
\mathbf{3 5} \mathbf{5}_{\mathrm{S}} \oplus \mathbf{3 5 _ { \mathrm { C } }}\end{array}$ & \\
\hline \multirow[b]{2}{*}{4} & $\mathrm{E}_{7(7)}$ & $\overline{\mathrm{SL}(2, \mathbb{R}) \times \mathrm{SO}(6,6)}$ & $\overline{(\mathrm{SL}(2, \mathbb{R}))^{3} \times \mathrm{SO}(4,4)}$ & $\overline{(\mathrm{SL}(2, \mathbb{R}))^{7}}$ \\
\hline & 8645 & $(1, \overline{462}) \begin{array}{r}(3,495) \\
(1,2079)\end{array}$ & $3 \times(\mathbf{3}, \mathbf{3 5}) \begin{array}{l}3 \times\left(\mathbf{3}^{2}, \mathbf{2 8}\right) \\
(\mathbf{1}, \mathbf{3 5 0})\end{array}$ & $16 \times 3^{3}$ \\
\hline \multirow[b]{2}{*}{3} & $E_{8(8)}$ & $\overline{\mathrm{SO}(8,8)}$ & 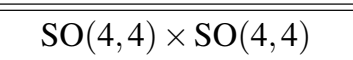 & $(\mathrm{SL}(2, \mathbb{R}))^{8}$ \\
\hline & 147250 & 643560060 & $\begin{array}{ll}4 \times(\mathbf{3 5}, \mathbf{3 5}) & (\mathbf{3 5 0}, \mathbf{2 8}) \\
(\mathbf{2 8}, \mathbf{3 5 0})\end{array}$ & $16 \times 3^{4}$ \\
\hline
\end{tabular}

Table 1: The representations of the space-filling branes that preserve the same supersymmetry of the truncation (in red) and those that are 1/2-BPS states (in black). In six dimensions the case with 16 supersymmetry is divided in two rows, with the upper row corresponding to the truncation to the $\mathscr{N}=(1,1)$ theory and the lower row to the truncation truncation to the $\mathscr{N}=(2,0)$ theory. In four and three dimensions, the representations of the cases with eight and four supersymmetry are written in a short-hand notation, see [2] for the details.

three truncations and the $\mathrm{O} 3$ truncation are degenerate, and therefore the branes that preserve the same supersymmetry are all the branes of the four truncations of the maximal theory together. This gives in total $3 \times 32=128$ branes, which turn out to be the long weights of the 16 representations of $S L(2, \mathbb{R})^{7}$, made of three triplets and four singlets, with the condition that there are either zero or two triplets with respect to the three $S L(2, \mathbb{R})$ 's associated to the $U_{i}$ moduli. As we mentioned in the introduction, these are precisely the representations of the tadpoles generated by the fluxes, that is the representations of the Bianchi identities of the $\mathscr{N}=2$ theory that survive the orientifold truncation but are not required by $\mathscr{N}=1$ supersymmetry.

\section{Gaugings $\&$ the embedding tensor}

Form the supergravity point of view, fluxes induce a gauging, which is described in terms of the embedding tensor $[19,20]$. If the ungauged theory has a global symmetry $G$, the embedding tensor belongs to a specific representation of $G$. Moreover, in general supersymmetry and gauge invariance impose an additional quadratic constraint on the embedding tensor, which requires that 
the product of two embedding tensors projected on specific representations of $G$ must vanish. The aim of this section is to consider $\mathbb{Z}_{2}$ truncations within gauged supergravities in various dimensions to obtain theories with less supersymmetries. The concrete prescription is to compare all the quadratic constraints required by the theory with more supersymmetry when restricted to its $\mathbb{Z}_{2}$-even sector with the ones imposed by the consistency of the theory with halved amount of supersymmetry. The mismatch between the two exactly identifies the irreducible representations containing space-filling branes allowed by the less supersymmetric theory. More precisely, we find that all the space-filling branes that preserve the same supersymmetry of the $\mathbb{Z}_{2}$ truncation belong to the representation of the symmetry of the truncated theory which is the highest-dimensional representation of the quadratic constraint which survives the truncation but is not required by the supersymmetry of the truncated theory.

We can give as an example the truncation to the half-maximal theory of maximal gauged supergravity in four dimensions. The most general maximal gauged theory was studied in [37] and it turns out to enjoy $E_{7(7)}$ global symmetry, with the embedding tensor belonging to the $\mathbf{9 1 2}$ of this group. Furthermore, in [21], the truncation defined by

$$
\begin{array}{ll}
\mathrm{E}_{7(7)} & \longrightarrow \mathrm{SL}(2, \mathbb{R}) \times \underbrace{\mathbf{9 1 2}}_{X_{\mathscr{M} N \mathscr{P}}} \underbrace{(\mathbf{2 , 1 2})}_{\xi_{\alpha M} \mathrm{SO}(6,6),} \oplus \underbrace{(\mathbf{2 , 2 2 0}, \mathbf{2 2 0})}_{f_{\alpha[M N P]}},
\end{array}
$$

was found to yield a half-maximal theory [38] coupled to six extra vector multiplets.

The quadratic constraints of the maximal theory

$$
\Omega^{\mathscr{M} \mathscr{Q}} X_{\mathscr{M} \mathscr{N} \mathscr{P}} X_{\mathscr{Q} \mathscr{R} S}=0,(\mathbf{1 3 3} \oplus \mathbf{8 6 4 5})
$$

give rise to the following quadratic constraints for $\mathbb{Z}_{2}$ even objects

$$
\begin{array}{rlrl}
\xi_{\alpha M} \xi_{\beta}{ }^{M} & =0, & & (\mathbf{3}, \mathbf{1}) \\
\xi_{(\alpha}{ }^{P} f_{\beta) M N P} & =0, & (\mathbf{3}, \mathbf{6 6}) \\
3 f_{\alpha R[M N} f_{\beta P Q]}{ }^{R}-2 f_{(\alpha[M N P} \xi_{\beta) Q]} & =0, & (\mathbf{3}, \mathbf{4 9 5}) \\
\varepsilon^{\alpha \beta}\left(\xi_{\alpha}{ }^{P} f_{\beta P M N}+\xi_{\alpha M} \xi_{\beta N}\right) & =0, & (\mathbf{1}, \mathbf{6 6}) \\
\varepsilon^{\alpha \beta} f_{\alpha M N R} f_{\beta P Q}{ }^{R}+(f \xi \text { terms }) & =0,(\mathbf{1 , 6 6}) \oplus(\mathbf{1}, \mathbf{2 0 7 9}) \\
f_{\alpha M N P} f_{\beta}{ }^{M N P} & =0, & (\mathbf{3}, \mathbf{1}) \\
\left.\varepsilon^{\alpha \beta} f_{\alpha[M N P} f_{\beta Q R S}\right|_{S D} & =0, & (\mathbf{1}, \overline{\mathbf{4 6 2}})
\end{array}
$$

where one can recognise all the quadratic constraints of $\mathscr{N}=4$ supergravity, plus the last two lines which therefore contain space-filling branes. In particular, the $(\mathbf{1}, \overline{\mathbf{4 6 2}})$ contains exactly the 32 long weights that we expect from the results of the previous section.

We find that exactly the same applies if one further truncates to theories with less supersymmetry. In particular, using the quadratic constraints of the embedding tensor of $\mathscr{N}=2$ theories discussed in [39], we find that the truncation of the four-dimensional $\mathscr{N}=2$ theory with symmetry $(\mathrm{SL}(2, \mathbb{R}))^{3} \times \mathrm{SO}(4,4)$ gives the $\mathscr{N}=1$ theory with $\mathrm{SL}(2, \mathbb{R})^{7}$ global symmetry. The embedding tensor is projected on the $(\mathbf{2}, \mathbf{2}, \mathbf{2}, \mathbf{2}, \mathbf{2}, \mathbf{2}, \mathbf{2})$ of $\operatorname{SL}(2, \mathbb{R})^{7}$, while the quadratic constraints are 
projected on the 16 representations made of three triplets and four singlets that are exactly the representations of the space-filling 3-branes that preserve the same supersymmetry of the truncation. Indeed, minimal supersymmetry does not require any quadratic constraint for the embedding tensor, and consistently we find that all the highest-dimensional representations of the quadratic constraints of the $\mathscr{N}=2$ theory that survive the $\mathbb{Z}_{2}$ truncation can be uplifted introducing spacefilling branes.

\section{Conclusions}

In this presentation we have reviewed the results of refs. [1, 2]. In particular, we have found a relation between the quadratic constrains of the embedding tensor and the space-filling branes that all preserve the same supersymmetry of the truncated theory. This explains how in the $T^{6} /\left[\mathbb{Z}_{2} \times \mathbb{Z}_{2}\right]$ IIB O3-O7 orientifold model the Bianchi identities of the $\mathscr{N}=2$ theory can be uplifted generating tadpoles for space-filling 3-branes. Crucial to the derivation of these results was the discovery of a universal T-duality rule for all the branes in string theory [17], which we give in eq. (2.5).

There is an elegant relation between these results and the structure of the supersymmetry algebra. The number of truncations to the theory with halved supersymmetry is always given by the number of vector central charges, which in turn is always equal to the different ways in which the global symmetry of the $\mathbb{Z}_{2}$ truncated theory can be embedded in the global symmetry of the original theory.

To conclude, what the results presented here show is that the inclusion of exotic branes may be an important tool in string compactifications with non-geometric fluxes. Indeed, it would enlarge the possibilities to find more viable $\mathscr{N}=1$ four-dimensional vacua where all moduli can be conveniently stabilised, as many constraints on the fluxes could be relaxed. Moreover, from a wider perspective which goes beyond the closed string sector, it could be of extreme interest to further extend this analysis studying the dynamics of the exotic branes.

\section{Acknowledgements}

I would like to thank the organisers of the 2018 workshop on "Dualities and Generalised Geometries" at the Corfu Summer Institute for giving me the opportunity to present these results.

\section{References}

[1] D. M. Lombardo, F. Riccioni and S. Risoli, "Non-geometric fluxes \& tadpole conditions for exotic branes," JHEP 1710 (2017) 134.

[2] G. Dibitetto, F. Riccioni and S. Risoli, “Space-filling branes \& gaugings," JHEP 1807 (2018) 006.

[3] M. Grana, "Flux compactifications in string theory: A Comprehensive review," Phys. Rept. 423 (2006) 91.

[4] S. Gukov, C. Vafa and E. Witten, "CFT's from Calabi-Yau four folds," Nucl. Phys. B 584 (2000) 69 Erratum: [Nucl. Phys. B 608 (2001) 477].

[5] G. Aldazabal, P. G. Cámara, A. Font and L. E. Ibáñez, "More dual fluxes and moduli fixing," JHEP 0605 (2006) 070. 
[6] J. Shelton, W. Taylor and B. Wecht, "Nongeometric flux compactifications," JHEP 0510 (2005) 085.

[7] A. Guarino and G. J. Weatherill, "Non-geometric flux vacua, S-duality and algebraic geometry," JHEP 0902 (2009) 042.

[8] A. Font, A. Guarino and J. M. Moreno, “Algebras and non-geometric flux vacua,” JHEP 0812 (2008) 050 .

[9] G. Aldazabal, P. G. Camara and J. A. Rosabal, "Flux algebra, Bianchi identities and Freed-Witten anomalies in F-theory compactifications,” Nucl. Phys. B 814 (2009) 21.

[10] C. Kounnas and M. Porrati, "Spontaneous Supersymmetry Breaking in String Theory,” Nucl. Phys. B 310 (1988) 355. doi:10.1016/0550-3213(88)90153-8.

[11] I. Antoniadis, E. Dudas and A. Sagnotti, "Supersymmetry breaking, open strings and M theory," Nucl. Phys. B 544 (1999) 469.

[12] I. Antoniadis, G. D’Appollonio, E. Dudas and A. Sagnotti, "Partial breaking of supersymmetry, open strings and M theory," Nucl. Phys. B 553 (1999) 133.

[13] I. Antoniadis, G. D’Appollonio, E. Dudas and A. Sagnotti, “Open descendants of Z(2) x Z(2) freely acting orbifolds," Nucl. Phys. B 565 (2000) 123.

[14] C. Angelantonj and A. Sagnotti, "Open strings,” Phys. Rept. 371 (2002) 1 Erratum: [Phys. Rept. 376 (2003) no.6, 407].

[15] B. Wecht, "Lectures on Nongeometric Flux Compactifications,” Class. Quant. Grav. 24 (2007) S773.

[16] E. A. Bergshoeff, V. A. Penas, F. Riccioni and S. Risoli, "Non-geometric fluxes and mixed-symmetry potentials," JHEP 1511 (2015) 020.

[17] D. M. Lombardo, F. Riccioni and S. Risoli, “P fluxes and exotic branes,” JHEP 1612 (2016) 114.

[18] G. Aldazabal, E. Andres, P. G. Camara and M. Grana, "U-dual fluxes and Generalized Geometry," JHEP 1011 (2010) 083.

[19] H. Nicolai and H. Samtleben, "Maximal gauged supergravity in three-dimensions," Phys. Rev. Lett. 86 (2001) 1686.

[20] B. de Wit, H. Samtleben and M. Trigiante, "On Lagrangians and gaugings of maximal supergravities," Nucl. Phys. B 655 (2003) 93.

[21] G. Dibitetto, A. Guarino and D. Roest, “How to halve maximal supergravity,” JHEP 1106 (2011) 030.

[22] J. de Boer and M. Shigemori, "Exotic branes and non-geometric backgrounds," Phys. Rev. Lett. 104 (2010) 251603.

[23] C. M. Hull, “A Geometry for non-geometric string backgrounds,” JHEP 0510 (2005) 065.

[24] E. Lozano-Tellechea and T. Ortin, “7-branes and higher Kaluza-Klein branes,” Nucl. Phys. B 607 (2001) 213.

[25] J. de Boer and M. Shigemori, "Exotic Branes in String Theory,” Phys. Rept. 532 (2013) 65.

[26] S. Elitzur, A. Giveon, D. Kutasov and E. Rabinovici, "Algebraic aspects of matrix theory on T**d," Nucl. Phys. B 509 (1998) 122.

[27] N. A. Obers, B. Pioline and E. Rabinovici, "M theory and U duality on $\mathrm{T}^{* *} \mathrm{~d}$ with gauge backgrounds,” Nucl. Phys. B 525 (1998) 163.

[28] N. A. Obers and B. Pioline, "U duality and M theory," Phys. Rept. 318 (1999) 113. 
[29] E. A. Bergshoeff and F. Riccioni, “D-Brane Wess-Zumino Terms and U-Duality,” JHEP 1011 (2010) 139.

[30] E. A. Bergshoeff and F. Riccioni, "String Solitons and T-duality,” JHEP 1105 (2011) 131.

[31] E. A. Bergshoeff, A. Marrani and F. Riccioni, “Brane orbits,” Nucl. Phys. B 861 (2012) 104.

[32] P. C. West, “E(11) and M theory,” Class. Quant. Grav. 18 (2001) 4443.

[33] E. A. Bergshoeff, F. Riccioni and L. Romano, "Branes, Weights and Central Charges," JHEP 1306 (2013) 019.

[34] B. R. Greene, A. D. Shapere, C. Vafa and S. T. Yau, "Stringy Cosmic Strings and Noncompact Calabi-Yau Manifolds,” Nucl. Phys. B 337 (1990) 1.

[35] E. A. Bergshoeff, J. Hartong, T. Ortin and D. Roest, “Seven-branes and Supersymmetry,” JHEP 0702 (2007) 003.

[36] A. Kleinschmidt, “Counting supersymmetric branes,” JHEP 1110 (2011) 144.

[37] B. de Wit, H. Samtleben and M. Trigiante, “The Maximal D=4 supergravities,” JHEP 0706 (2007) 049.

[38] J. Schon and M. Weidner, “Gauged N=4 supergravities,” JHEP 0605 (2006) 034.

[39] B. de Wit, H. Samtleben and M. Trigiante, "Magnetic charges in local field theory," JHEP 0509 (2005) 016. 